\title{
Towards the concept of disease-modifier in post-stroke or vascular cognitive impairment: a consensus report
}

\author{
Régis Bordet ${ }^{1,8^{*}}$, Ralf Ihl$l^{2}$, Amos D. Korczyn ${ }^{3}$, Giuseppe Lanza ${ }^{4}$, Jelka Jansa ${ }^{5}$, Robert Hoerr ${ }^{6}$ and Alla Guekht ${ }^{7}$
}

\begin{abstract}
Background: Vascular cognitive impairment (VCl) is a complex spectrum encompassing post-stroke cognitive impairment (PSCl) and small vessel disease-related cognitive impairment. Despite the growing health, social, and economic burden of $\mathrm{VCl}$, to date, no specific treatment is available, prompting the introduction of the concept of a disease modifier.

Consensus and suggestions: Within this clinical spectrum, $\mathrm{VCl}$ and $\mathrm{PSCl}$ remain advancing conditions as neurodegenerative diseases with progression of both vascular and degenerative lesions accounting for cognitive decline. Disease-modifying strategies should integrate both pharmacological and non-pharmacological multimodal approaches, with pleiotropic effects targeting (1) endothelial and brain-blood barrier dysfunction; (2) neuronal death and axonal loss; (3) cerebral plasticity and compensatory mechanisms; and (4) degenerative-related protein misfolding. Moreover, pharmacological and non-pharmacological treatment in PSCl or VCl requires valid study designs clearly stating the definition of basic methodological issues, such as the instruments that should be used to measure eventual changes, the biomarker-based stratification of participants to be investigated, and statistical tests, as well as the inclusion and exclusion criteria that should be applied.
\end{abstract}

Conclusion: A consensus emerged to propose the development of a disease-modifying strategy in $\mathrm{VCl}$ and PSCl based on pleiotropic pharmacological and non-pharmacological approaches.

Keywords: Disease-modifying therapy, Post-stroke cognitive impairment, Vascular cognitive impairment, Vascular dementia, Clinical trial, Multimodal approach

\section{Background}

Given the aging population, increased prevalence of cognitive impairment and dementia in the coming decades has been likened to a "tsunami", due to the human, social, and economic consequences they will engender [1]. Cerebral or systemic vascular diseases play a relevant role in this "tsunami". Vascular cognitive impairment (VCI) leading to vascular dementia (VaD) is a clinical spectrum that can be related either to an acute event, namely a stroke (i.e., post-stroke cognitive impairment; PSCI), or to progressive vascular lesions (leukoaraiosis,

\footnotetext{
*Correspondence: regis.bordet@univ-lille2.fr

'University of Lille, Inserm, CHU, U1171 'Degenerative and vascular cognitive disorders', Lille, France

${ }^{8}$ Département de Pharmacologie Médicale, Faculté de Médecine, 1 place

Verdun, 59045 Lille Cedex, France

Full list of author information is available at the end of the article
}

Binswanger leukoencephalopathy, white matter lesions, microbleeds) related to small vessel disease. Post-stroke $\mathrm{VaD}$ and $\mathrm{VaD}$ itself were the first presentations to be recognized. $\mathrm{VaD}$ was initially represented by multi-infarct dementia, although the concept was then expanded to subcortical ischemic VaD, strategic-infarct dementia, hypoperfusion dementia, and hemorrhagic dementia. In a second step, the existence of mixed Alzheimer's disease (AD) and $\mathrm{VaD}$ was recognized [2]. More recently, cognitive decline has been identified to exist even prior to the stage of overt dementia, and mild cognitive impairment related to vascular lesions has been identified as the preferred target of therapeutic strategies in order to slow or stop the decline, thus avoiding progression towards dementia and related loss of autonomy in daily living.

All clinical aspects of VCI share the frequent association with vascular risk factors or systemic vascular 
diseases that lead patients to be prone to developing large or small artery remodeling, explaining the occurrence of vascular brain lesions. The scenario is more complex when considering the potential direct impact of vascular or metabolic risk factors on cognition and the interaction between vascular load and neurodegenerative lesions such as AD-related pathology [3, 4] (Fig. 1). Frailty syndrome could be also a contributor to this complexity [5]. Cognitive dysfunction and dementia frequently occur following an acute stroke, and they are an important cause of stroke-related morbidity. Dementia may be related to a pure $\mathrm{VaD}$ or to a mixed form, which occurs after a stroke, or can represent the progression of pre-stroke vascular or degenerative-related cognitive impairment [6] (Fig. 2). There is a large variability in terms of manifestations of cognitive decline in a vascular context, and a delay in the diagnosis when there are no evident or acute symptoms is often observed. The occurrence of an acute event, such as a stroke, provides unique opportunities to follow cognitive function and for the early detection of VCI from a preventive or therapeutic perspective. Indeed, prevention and treatment of PSCI and VCI are the critical priorities for both clinical care and scientific research. The growing health, social, and economic burden of PSCI or VCI is driving the demand for clinical studies that evaluate the benefits and risks of both pharmacological and non-pharmacological interventions [7]. A better understanding of the risk factors and an estimation of the risk scores for PSCI or VCI are of crucial importance for the selection of patients and the design of preventive clinical trials.

Despite the presence of an initial acute event, both VCI and PSCI remain pathodynamic conditions, as neurodegenerative diseases, since there is a progression of both vascular and degenerative lesions accounting for cognitive decline [8]. A prodromal phase might be represented by the period when vascular or metabolic risk factors induce silent brain lesions or by the frailty syndrome, which has been recently considered as a prodromal of $\mathrm{VaD}$ [9]. In terms of treatment, research has focused largely on the acute phase of vascular lesions rather than their progression, whereas the main issue remains related to the combination of both vascular and degenerative factors that overlap, despite exhibiting distinctive pathophysiological mechanisms [10]. At the present time, the proposal of the concept of a disease modifier within the spectrum of $\mathrm{VaD}$ is warranted. Nevertheless, several issues should be addressed to make this concept operant in its specific field given the complexity of this medical spectrum in terms of evolution (occurrence or not of an acute event), cognitive phenotype, lesion burden, and pathophysiology (pure or mixed lesions). It is also necessary to highlight the differences and similarities with the concept of a disease modifier in the context of neurodegenerative diseases. A diseasemodifying strategy, defined as a therapeutic approach aiming to change the natural course of an illness, applies primarily to chronic diseases. In the field of neurological disorders, this concept has been used for neurodegenerative and neuroinflammatory diseases. The mechanisms involved in these conditions are multiple, and therapeutic options are not limited to a single neuroprotection but are integrated in a multimodal approach, including anti-inflammatory, antioxidants or vascular mechanisms, as well as the modulation of protein aggregation and synaptic or neuronal plasticity $[11,12]$. Along with neurodegenerative diseases, PSCI and VCI share a preclinical phase prior to the progressive appearance of the first symptoms or after an acute event such as a stroke. PSCI and VCI also resemble neuroinflammatory diseases that show the peculiarity, in their remittent form, to couple the occurrence of acute episodes with a

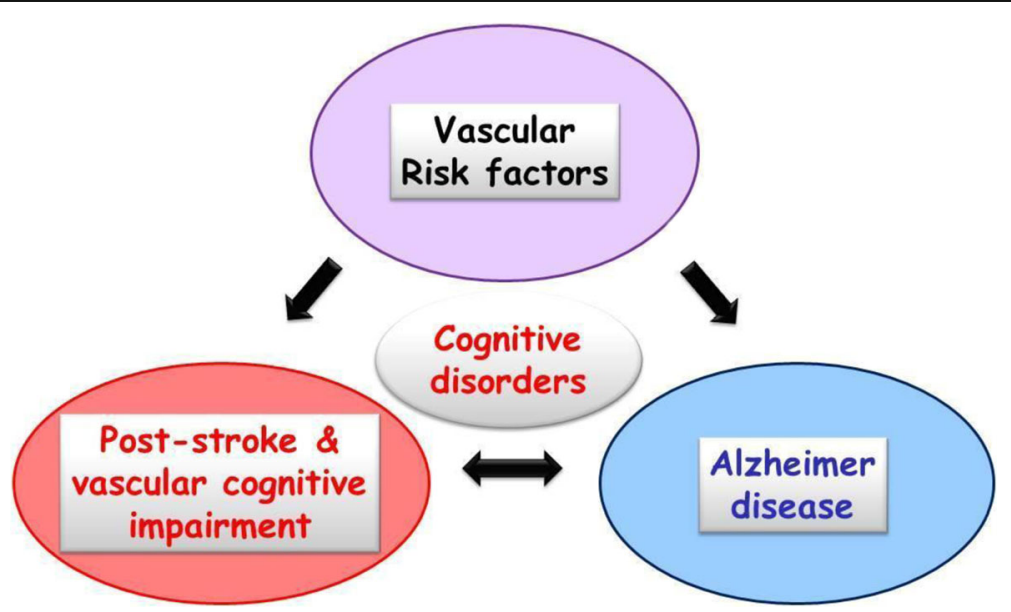

Fig. 1 The crossroad of cognitive disorders. Cognitive disorders may be related to vascular or degenerative processes, or both in the mixed form. Vascular risk factors influence both vascular- and degenerative-related cognitive impairment, but also have a direct impact on cognitive functions 


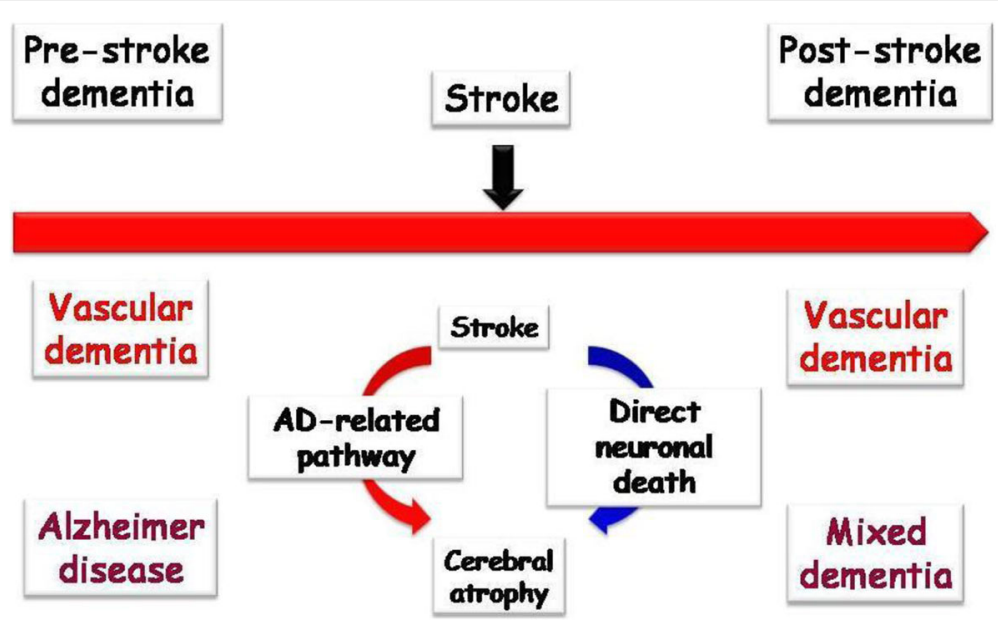

Fig. 2 Interactions between pre- and post-stroke cognitive disorders and dementia. Pre-stroke dementia may be related to vascular or degenerative processes, indicating that cognitive impairment after a stroke can be associated with a pure vascular mechanism or a mixed process. Stroke induces regional atrophy through neuronal death directly related to the vascular lesion, or indirectly to the Alzheimer's disease (AD)-related pathway

gradual evolution to functional deficits, especially related to motor and cognitive manifestations. Therefore, the natural clinical history suggests that the concept of a disease-modifying effect is applicable to PSCI and VCI, as well as in neurodegenerative or neuroinflammatory pathologies. Moreover, particularly in older patients, VCI or PSCI are rarely "pure" but more often combined with AD-type lesions that contribute to the evolution and justify a disease-modifying strategy [6].

When embarking on trials of potentially diseasemodifying treatments for VCI, a look at what we have learnt from disease modification and prevention trials in the neighboring field of $\mathrm{AD}$ is commendable. Assuming that the $\beta$-amyloid $(A \beta)$ pathology is causally related to dementia in AD, anti-amyloid treatments (e.g., $\gamma$-secretase inhibitors, monoclonal antibodies) have been considered as disease-modifying agents par excellence, although a close relationship between amyloid and cognition has not been well established [13]. After a number of failed clinical trials, the role of amyloid is being re-considered, although its feasibility as a therapeutic target has become questionable; even inhibition of $\gamma$-secretase led to a faster cognitive and functional decline, probably due to the involvement of this enzyme in other biochemical pathways such as in notch signaling [14]. Anti-A $\beta$ immune therapy is still being tried in mild to moderate AD. However, positron emission tomography studies have shown that amyloid deposition mostly takes place before the dementia stage of $\mathrm{AD}$ and reaches a plateau once overt dementia is present [15]. $A \beta$ is therefore still considered by some to be the right target, but the dementia stage of the disease may be beyond the window of opportunity. Moreover, amyloid imaging sub-studies of two bapineuzumab phase-III trials found that, in $6.5 \%$ of Apolipoprotein E $\varepsilon 4$ carriers and in
$36 \%$ of non-carriers, the cortical amyloid load was below the specified threshold for amyloid positivity (i.e., the therapeutic target was missing) [16]. Similar rates of patients with sparse or lacking amyloid plaques were found in an autopsy trial involving 200 patients with a primary diagnosis of $\mathrm{AD}$ [17]. In a number of trials testing the disease-modifying potential of treatments, progression to dementia was chosen as the primary outcome (e.g., [18]). To date, the exact determination of the temporal point at which, during the course of an ongoing disease, a specific threshold is crossed has remained problematic [19].

The multifactorial pathogenesis of PSCI and VCI needs to consider drug combinations or multimodal agents to change the course of the disease, as well as the search of selective ligands targeting distinctive cellular or molecular pathways. Beyond pharmacological agents, non-pharmacological approaches might also be included in this scenario. Nevertheless, potential pitfalls should be taken into consideration, including (1) addressing the wrong target, (2) interfering with the target pathology outside the window of opportunity, (3) patients lacking the target pathology, and (4) choosing insensitive outcomes.

The main objective of this paper is to demonstrate the relevance of a disease-modifying multimodal strategy based on the known pathophysiological pathways and to propose a model of preclinical and clinical development. Even though PSCI forms part of the VCI spectrum, the two entities are distinguished throughout since the different onset type for the two clinical conditions is able to induce a difference in the assessment of the disease-modifying strategy.

\section{Methodology}

A focus meeting on disease modifiers in VCI was held as a part of the "9th International Congress on Vascular 
Dementia" in Ljubjana, Slovenia, in October 2015. Experts in this field attending the meeting reviewed the current evidence and literature data. For the purpose of this topic review, a comprehensive search on Medline, PubMed, and Embase databases for studies published until April 30, 2016, was conducted. The keywords used in the current search were disease modifier, disease-modifying drug, disease-modifying therapy, disease-modifying strategy, post-stroke cognitive disorder, post-stroke dementia, vascular cognitive impairment, and vascular dementia. Following review of all relevant abstracts, few articles were related to the topic of the paper, prompting the panel experts to define, by themselves, the concept and to highlight the main themes enriching the concept, namely the pathophysiology and pharmacological targets, the potential role of existing drugs, the interest of non-pharmacological approaches, and the need for a specific methodology for future clinical trials. A proposal on disease modifiers in PSCI and VCI was drafted and reviewed by the group. The draft was repeatedly circulated and discussed before being finalized.

\section{From pathophysiological mechanisms to pharmacological targets for emerging strategies}

The association of stroke and dementia is frequent and can be seen either in the diagnostic work-up of patients attending a memory clinic or during the follow-up of stroke survivors $[6,20,21]$. Both ischemic and hemorrhagic strokes lead to a high risk of cognitive impairment and dementia $[6,20,22]$. About one in ten patients has dementia prior to the first stroke, one in ten develops new dementia after the first stroke, and more than one in three develops dementia after a shortly recurrent stroke [23]. A genetic susceptibility has been identified through several gene polymorphisms associated with PSCI or VCI occurrence, in particular the Apolipoprotein E polymorphism [24].

Animal experimental studies on mechanisms and predictors of post-stroke dementia might provide keys for preventive and disease-modifying treatment. Many animal models have been developed to identify some mechanisms of action, including (1) long-term follow-up of middle cerebral artery occlusion; (2) transgenic mice reproducing certain genetic diseases associating stroke and cognitive impairment such as cerebral autosomal dominant arteriopathy with subcortical infarcts and leukoencephalopathy (CADASIL); (3) long-term models of risk factors or bilateral carotid stenosis; and (4) combination of vascular and degenerative lesions in transgenic mice $[25,26]$. The assessment of these models combines behavioral tasks, neuroimaging, and cellular and molecular explorations. A recent review emphasizes the role of different animal models in VCI, concluding that no model is able to completely replicate the clinical and pathologic aspects of VCI spectrum explaining, at least in part, the gap between experimental results and translation to clinical reality [27]. In addition, models mimic lesions rather than cognitive decline, with a major limitation in terms of follow-up duration. The development of long-term models should be a priority to improve the relevance of animal experiments. In addition, new approaches should be tested for pharmacological studies in several models to bring more convincing results before a translation to the clinical steps.

International cohort-based consortia (Strokog or Metacohort) are sharing data on biomarkers (genetic, biological, neuroimaging) sampled in stroke patient cohorts with longterm follow-up to assess cognitive evolution [28, 29]. For example, the STROKDEM (Study of Factors Influencing Post-stroke Dementia; NCT01330160) and DEDEMAS (Determinants of Dementia After Stroke) studies are looking at biomarkers (neuroimaging, biological or genetic markers, endothelial function-derived factors) and interactions between vascular and neurodegenerative mechanisms, with an emphasis on the impact of secondary prevention treatments [30]. The Tel-Aviv Brain Acute Stroke Cohort (TABASCO) trial, with up to 10 years follow-up after stroke, is focused on the association between predefined demographic, psychological, inflammatory, biochemical, neuroimaging, and genetic markers measured during the acute phase [31].

The relationship between stroke and cognitive disorders remains complex given that a stroke may induce cognitive impairment through several mechanisms that are often cumulative or synergistic; for example, (1) vascular brain lesions (infarcts or hemorrhages) can be symptomatic by themselves through a location in a strategic area in terms of cognitive functioning; (2) previous silent vascular lesions (leukoaraiosis, Binswanger leukoencephalopathy, microbleeds, silent infarcts, silent hemorrhages, atrophy) may contribute to the burden responsible for cognitive impairment through a cumulative effect or dysconnectivity; (3) accelerated evolution of pre-existing degenerative lesions through hypoxia mechanism; (4) direct effect of vascular or metabolic risk factors associated with stroke occurrence on cognitive functioning; (5) direct induction of neurodegeneration responsible for global or regional brain atrophy; (6) impairment of endothelium function and blood-brain barrier leakage; and (7) induction of neuroinflammation [32, 33] (Fig. 3).

A better understanding of the cellular and molecular processes involved in the dysfunctional neurovascular unit may lead to improved treatments for both PSCI and VCI. Increased attention is being paid to anti-inflammatory strategies, as suggested by the extension and persistence of neuroinflammation after a stroke that may interact with pre-existing $\mathrm{AD}$ pathology and accelerate neurodegeneration [34]. Oxidative stress remains an important pathway involved in both neuronal and endothelial injury [35]. 


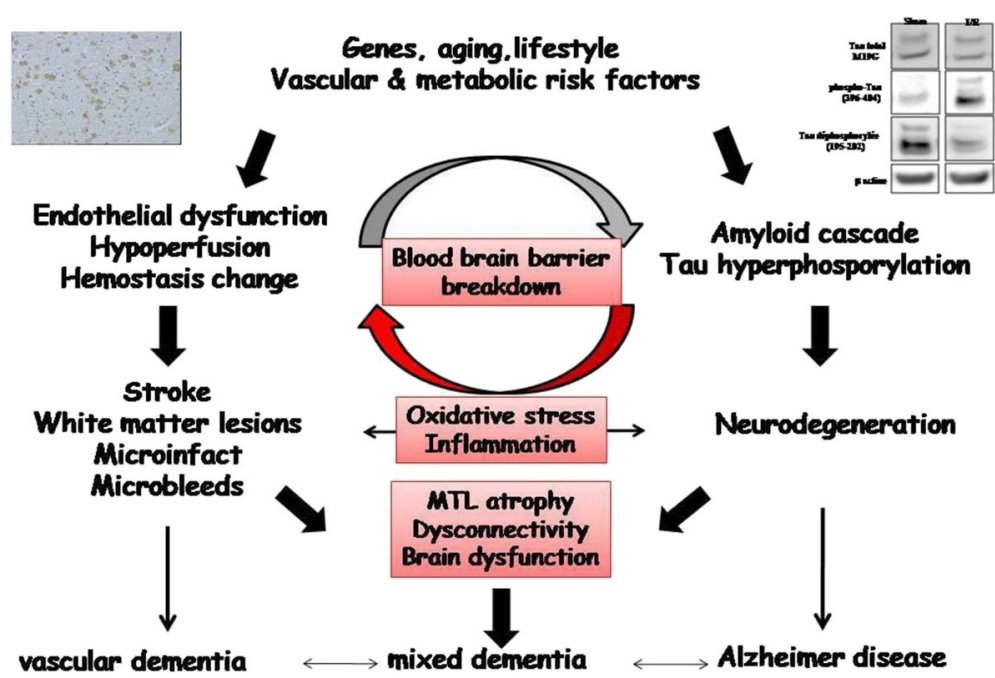

Fig. 3 Pathophysiological mechanisms of interactions between vascular and degenerative processes in both vascular and mixed dementia (Adapted from [40]). MTL medial temporal lobe. The figure integrates an image of western blot (tau expression after middle cerebral artery occlusion in mice) and immunohistochemistry (amyloid peptide expression after middle cerebral artery occlusion in rat)

Neurovascular uncoupling is also responsible for disturbance of brain oxygenation and vascular reactivity necessary to supply sufficient blood flow in response to neuronal metabolism [36]. Neurohormonal pathways, changes in brain plasticity or neurotrophic factors, ion channels and mitochondrial dysfunction have all been observed in PSCI [37-39]. Stroke is also able to interact with the specific pathophysiology of neurodegenerative pathways (amyloid or tau cascades), as vascular or metabolic factors modulate the amyloid or tau cascades associated to $\mathrm{AD}[32,40]$. In contrast, amyloid lesions co-existing with VCI are associated with a greater cognitive dysfunction [41]. The cognitive deterioration is also related to an impairment of neurotransmission pathways, in particular cholinergic or glutamate transmission. More animal and clinical studies are needed to better characterize all the molecular mechanisms contributing to neuronal damage in PSCI and VCI as well as pharmacological targets aiming to develop a disease-modifying therapy with pleiotropic compounds or multimodal combinations targeting (1) endothelial function and blood-brain barrier; (2) neuronal death; (3) cerebral plasticity and compensatory mechanism; or (4) degenerative disease-related protein misfolding (Fig. 4).

\section{Is there a place for existing drugs?}

As demonstrated by the long history of neuroprotection in stroke and the failure of development of new compounds in $\mathrm{AD}$, the search of new drugs remains challenging, thus explaining the ongoing research to find additional properties to drugs currently in use or the development of new indications for existing drugs and fitting with relevant targets for PSCI or VCI [7, 42, 43].
The strategy of drug repurposing would be more successful if the assessed drugs were used in a disease or a risk factor closely related to PSCI or VCI. In this context, the focusing of symptomatic drugs on the treatment of cognitive or behavioral symptoms could also be of interest through additional mechanisms related to disease-modifying effects. Beyond pharmacological options, dietary supplements might be also considered in this regard if they target one or more relevant pathways as, for example, omega-3 [44].

Currently available symptomatic drugs are of interest since evidence-based data in controlled clinical trials have demonstrated the beneficial effect of acetylcholinesterase inhibitors and memantine administration to improve cognition, behavior, and activities of daily living in some patients $[45,46]$. Memantine has a lower evidencebased impact but is well tolerated, improves function, and reduces care dependency in treated patients compared to placebo [47]. The size of the effect remains low but is not negligible for patient care to decrease aggressivity or apathy, even though some guidelines do not recommend its general use [42, 47]. Nevertheless, the use of symptomatic drugs as and disease-modifying drugs should not be considered antagonistic, since patients require improvement of a complementary symptomatic effect in addition to the retardation of a disease not immediately detected by the patients or their carers. Inhibitors of acetylcholinesterase or memantine should participate in a multimodal strategy, particularly since they have experimentally demonstrated specific pharmacological effects on certain cellular and molecular pathways involved in PSCI or VCI [39]. The same holds true with antidepressant drugs and omega-3 fatty acid, both of which could act on mood (which is 


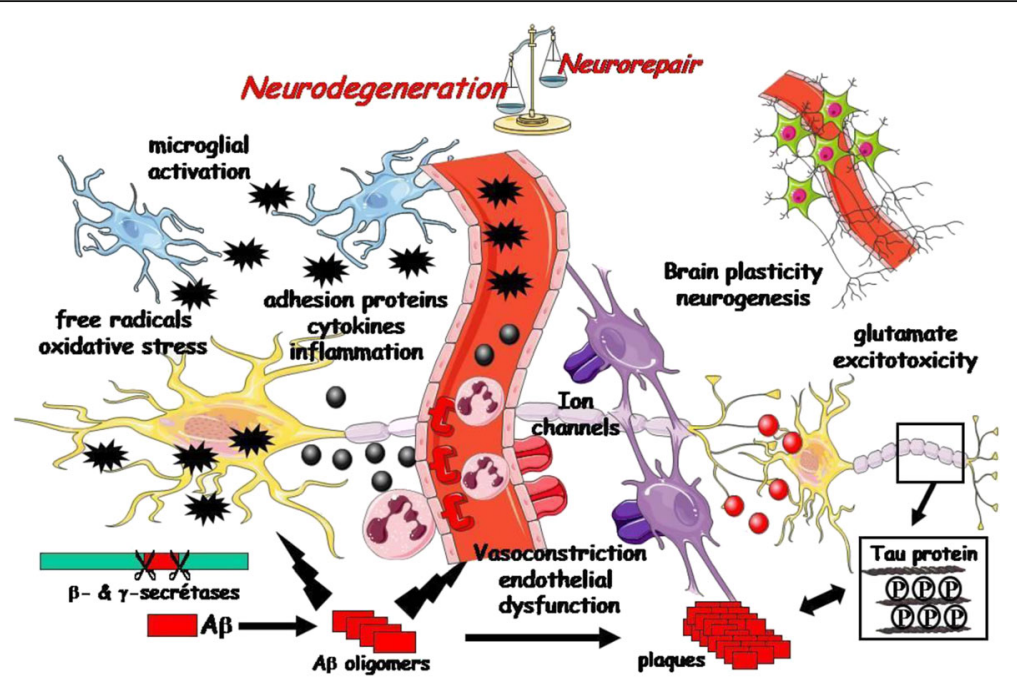

Fig. 4 Cellular and molecular mechanisms of vascular dementia and post-stroke cognitive disorders: presentation of all pathways involved in the neurovascular unit and their contribution to vascular impairment and neuronal injury responsible for occurrence and progression of cognitive decline. $A \beta$ beta-amyloid

often impaired in such patients) and brain plasticity or neurotrophic factors [48].

The treatment of vascular risk factors offers obvious possibilities for the prevention and impact on disease progression. Obesity, metabolic syndrome, physical inactivity, hypertension, hypercholesterolemia, and other vascular risk factors are associated with cognitive decline and dementia. The relationship between diabetes, stroke, and dementia is also well known [31, 49-52]. Compared with the general population, people with type 2 diabetes have a 1.5- to 2.5-fold increased risk of dementia and, to date, one in 10 to 15 cases of dementia can be attributed to type 2 diabetes [53]. At the cellular level, type 2 diabetes is associated with mitochondrial dysfunction, endoplasmic reticulum stress, increased inflammation, and altered energy metabolism. Anti-diabetic drugs act on hyperglycemia, dyslipidemia, and insulin resistance, and can counteract associated tissue inflammation. A number of anti-diabetic drugs, such as metformin, thiazolidinediones, and compounds targeting the glucagon-like peptide-1 receptor, are able to influence brain metabolism, neuroinflammation, and regeneration. These drugs could be developed as disease-modifying therapies for human brain diseases in patients with and without diabetes [54-56]. The impact of lipid-lowering drugs remains controversial, even though statins and fibrates have demonstrated anti-inflammatory, anti-oxidant, and endothelial properties as well as the ability to modulate brain plasticity through stem cell endogenous mobilization or the ADrelated amyloid cascade [7, 57-59]. All anti-hypertensive drug classes have demonstrated similar impact on cellular and molecular pathways. Pharmaco-epidemiological data also suggest that the use of anti-hypertensive drugs is associated with prevention or delayed occurrence of dementia, even though the evidence-based demonstration remains limited to few old clinical trials. Recent metaanalysis pointed out the interest of renin-angiotensin system modulation with both inhibitors of angiotensin converting enzyme and antagonist of angiotensin-2 receptors [60]. The combination of several drug classes could produce a higher effect, with variability of response in function of the etiology of stroke or vascular lesions. This potential favorable impact might be explained by the action of these drugs on the super-family of nuclear receptors, the so-called peroxisome proliferator-activated receptor, which have been demonstrated to exert pleiotropic effects within the brain [61]. Profiling drugs for vascular or metabolic risk factor treatment might be helpful in the selection of more interesting molecules primarily used in the secondary prevention after a stroke.

There is increasing evidence that alterations within the brain neurotrophic support, in particular the brainderived neurotrophic factor (BDNF) and the nerve growth factor (NGF) expression and signaling, may contribute to neurodegeneration. Indeed, one of the promising strategies of the modifying therapies might be associated with the use of specific neurotrophic factors as well as their modulation by antidepressant or omega3 fatty acid agents $[62,63]$. For instance, cerebrolysin is a peptide preparation with neurotrophic activity demonstrated in different in vitro and in vivo models; its mechanism of action might involve the modulation of the pro-NGF/NGF balance and a concomitant protection of cholinergic neurons [64]. The results of the large, multicenter, double-blind, placebo-controlled study in $\mathrm{VaD}$ demonstrated that the drug significantly improved the 
clinical outcome in $\mathrm{VaD}$ patients; moreover, it was safe and well tolerated. The Cochrane review stated that cerebrolysin may have positive effects on both cognitive and global functioning in elderly patients with $\mathrm{VaD}$ of mild to moderate severity [65-67]. Actovegin, a deproteinized ultrafiltrate of calf blood comprising more than 200 bioactive constituents, exhibits a range of pleiotropic neuroprotective and metabolic effects [68-70]; a study in a rat model of transient global cerebral ischemia found that it improved spatial learning and memory [70]. The recently completed ARTEMIDA study showed that actovegin $(2000 \mathrm{mg}$ i.v. solution for up to 20 daily infusions followed by $1200 \mathrm{mg} /$ day orally for the 6month remaining period) improved cognitive outcomes in patients with PSCI compared to placebo [71, 72]. Recent pre-clinical studies on Ginkgo biloba extract EGb 761 demonstrated that it improves mitochondrial function and energy metabolism, promotes hippocampal neurogenesis and plasticity, and enhances cerebral blood flow by decreasing blood viscosity [73, 74]. This multifactorial pharmacodynamic profile associated with safety and some symptomatic benefits in clinical studies of dementia [75-77] might provide promising signals for the possible disease-modifying potential that need to be investigated in further clinical trials.

\section{Is there an evidence of the disease-modifying effect of the non-pharmacological strategies?}

Repetitive transcranial magnetic stimulation (rTMS) and transcranial direct current stimulation (tDCS) are noninvasive and painless brain stimulation techniques able not only to explore cortical circuits and related neurochemical pathways in dementing illnesses but also to induce cortical plasticity with potential therapeutic and rehabilitative purposes [78, 79]. Several studies, although methodologically heterogeneous and most of them open-label in design, have shown that specific paradigms of stimulation might improve cognitive performance, thus possibly becoming an alternative to conventional neuroleptic therapy for psychiatric symptoms of dementia [79]. In $\mathrm{AD}$, these effects are probably mediated by compensatory mechanisms supporting the residual abilities [80], and the efficacy can be maximized by selecting patients on the basis of putative neurophysiological markers [78]. Although less is known, similar plastic phenomena are invoked in $\mathrm{VaD}$ [81]. High frequency rTMS over the left dorsolateral prefrontal cortex improved executive performance in patients with subcortical ischemic vascular disease, and the effect has been hypothesized to be due to an indirect activation of dopaminergic neurons in the midbrain and the noradrenergic and serotonergic neurons in the brainstem [82]. Moreover, rTMS is also possibly effective in alleviating symptoms of vascular depression [83]. More recently, the restorative effects of these techniques on cognitive ability have been observed in a murine model of $\mathrm{VaD}$, possibly through the neurotrophin release (such as the BDNF) and the induction of hippocampal NMDAmediated synaptic plasticity [84, 85]. Summarizing, preliminary results revealed that rTMS and tDCS can induce beneficial effects on specific cognitive domains and neuropsychiatric manifestations, although there are limited data and their clinical significance needs to be further validated. Major challenges exist in terms of appropriate patient selection and optimization of the stimulation protocols.

Alternative non-pharmacological strategies identified from traditional medicine should be considered. A systematic review tested the interest of Chinese herbal medicine as an adjunctive and even though some data suggest that this approach could improve cognitive impairment and enhance immediate response and quality of life in VCI patients, limitations of methodological quality warn to improve the design of clinical studies $[86,87]$. A recent meta-analysis also suggests a potential effect of acupuncture, but a better definition of trial design is mandatory $[88,89]$.

Considering the fact that $\mathrm{VaD}$ may be associated with a history of stroke with residual motor, perceptual, and cognitive deficits, patients should also have an access to multidisciplinary neurorehabilitation. Further, physiotherapy and occupational therapy (OT) should be based on the up-to-date evidence and consider the impact of cognitive problems on walking ability, falls, and daily functioning [90]. Physical activity may have a significant positive effect on cognitive functioning and ability to perform activities of daily living in the elderly, including patients with both degenerative and vascular dementia [91]. Both the individual and group physical interventions of different types, such as walking, gait and balance training, endurance training, and aerobic-based exercises, have been promising to delay the progression of cognitive decline and to improve physical and mental health $[92,93]$. The exercise routine, however, should be based on patient preferences and adjusted to their capabilities. The emerging evidence coming from neurorobotics and exergaming technology (video games) also provide encouraging results, especially because they enhance the adherence to rehabilitation interventions. Accelerometers are becoming an important method of physical assessment as they can be used in real life situations.

Recent evidence coming from Parkinson's disease and healthy aging population studies suggests that engagement in non-exercise physical activity (including involvement in any meaningful occupation) may also have an independent effect on functional performance [94, 95]. $\mathrm{VaD}$ typically leads to impairments in the performance of daily occupations, a key focus of the OT intervention 
process, and therefore patients with $\mathrm{VaD}$ are commonly referred to OT. Within the OT process, the activities of daily living, patient life roles, participation in enjoyed hobbies, and social, recreational, and leisure activities are explored [96]. The underlying reasoning for an effective performance of patients with $\mathrm{VaD}$ could be based on the Person-Environment-Occupation model, relying on the degree of fit between person, environment, and occupation. Occupational challenges occur when there is a poor congruence among a person's capacity, demands of the chosen occupation, and environmental supports and barriers [97]. Assessment results, based on the observation of actual occupational performance, have been shown to be a better indicator of a patient's ability to resume independent living than assessment of the level of impairment alone (such as the assessment of executive function, apraxia, apathy, and others) [98]. Moreover, it has been shown that OT improved daily functioning of people with dementia and reduced the burden on the caregiver, despite the patient's limited learning ability [99]. In addition, it has been reported that the quality of the relationship between the person with dementia and their caregiver is an important predictor of whether a patient with dementia will stay in the community or need to enter an institution for ongoing care [100]. To promote well-being, patient choices will be promoted and encouraged, and new hobbies introduced, relevant to an individual's living context. Examples of leisure activities may include horticultural therapy, regular social and recreational activities, Tai Chi [101], music therapy
[102], and art therapy. However, further evidence for their efficacy is needed.

In conclusion, PSCI and VCI patients might benefit from ongoing and collaborative working between pharmacological and non-pharmacological treatments, including rTMS, tDCS, cognitive training, and exercise and nonexercise physical activities to introduce appropriate support according to changes in functional needs and prevention or treatment of vascular risk factors. An effective multimodal intervention process makes it possible to improve, sustain, and even delay the decline in overall functional performance of people with PSCI or VCI. The assessment of daily occupations should be further evolved to provide detailed information about the deficits in activities of daily living and consequently improve treatment interventions.

\section{What development process should be proposed?}

In the near future, the main challenge will be to propose a way to develop new or repurposing drugs, alone or in combination with either a pharmacological or nonpharmacological approach, in PSCI or VCI. The crucial step of this challenge will take place in preclinical or clinical early phases before Go/No Go decision to continue into phase 3 . At this stage, a matrix approach should be used, including multiple animal models, healthy volunteers, and a stratified patient population, with several sources of assessment through different clinical, neurophysiological, or imaging biomarkers (Fig. 5). A specific overview of the types of failures in clinical trials can be

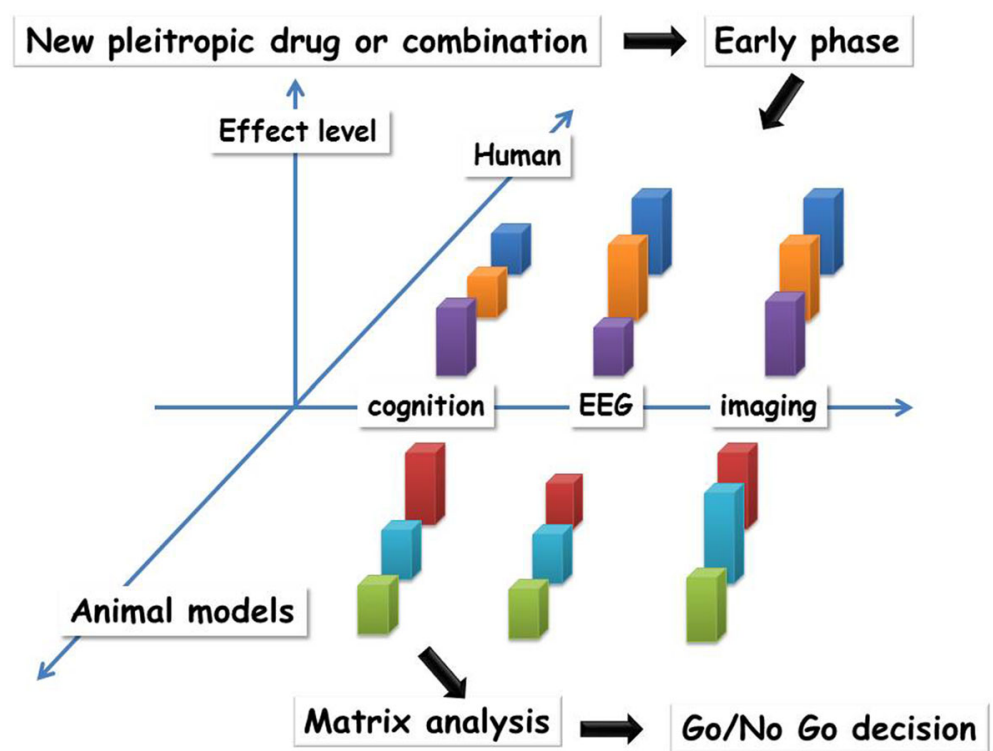

Fig. 5 Early phases of strategy development through a matrix approach using a biomarker battery. The aim of this process is to reinforce the rationale of Go/No Go decision before the translation to phase 3. For each animal or clinical study, the effect level should be determined and integrated into a matrix model in order to properly assess the relevance of the tested strategy 
found in the World Federation of Societies of Biological Psychiatry guidelines on dementia treatment, with an emphasis on the failures that are preventable [103]. In this context, a group of researchers has recently published a guideline to support investigators in developing the design of studies; they have identified several issues to be addressed, namely clarifying the research question, describing dropouts and survival, selecting appropriate study participation, practice effects, reliability, and unequal interval scaling, specifying the time scale, the non-linearity development of the severity of cognitive symptom, the time varying exposures and confounding, and the false discovery and over-fitting [104].

The choice of tested strategies should be driven by pathophysiological targets with relevant combinations to exert pleiotropic and multimodal effects. Preparing this choice will start with a systematic literature search and the definition of a preclinical and clinical study design. When a literature search is performed, caution has to be taken to avoid misleading conclusions. For meta-analyses and reviews, Rosenthal and De Matteo [105] outlined the advantages and disadvantages. Moreover, it should be kept in mind that the peer-review system does not always guarantee scientific excellence [106]. Moreover, to prove the details of studies, reviews and meta-analyses are not always useful.

Valid study designs are needed for both pharmacological and non-pharmacological treatment of PSCI or VCI, by defining basic methodological issues, such as the instruments that should be used to measure eventual changes, the number of participants to investigate, the statistical tests to be used, and the inclusion and exclusion criteria to be defined. Moreover, the experimental design should also be addressed; the standard design is a randomized, double blind, placebo-controlled study that aims to demonstrate improvement in at least one symptom, although this is not always considered the best solution, in particular because of the natural history of the disease or ethical reasons [107]. An answer to this question might come from statistics, by calculating the number needed to treat [108] or the real probability of an outcome. This underlines the need of including adequate statistical knowledge in the preparation of a study design. Additionally, special challenges have to be taken into account, such as the different possible diagnostic definitions of $\mathrm{VaD}$ spectrum, the co-morbid diseases, and the high number of possible intervening variables. Here, an overview of possible pitfalls when creating study designs has been provided and conclusions on more improved designs derived. Investigations in $\mathrm{VaD}$ bear a higher potential of methodological pitfalls than studies in most of other dementias since both the causes and course of $\mathrm{VaD}$ are heterogeneous and, in most cases, not predictable, leading to a high variability in what needs to be investigated. This heterogeneity means the need to develop clinical, biological, functional, and lesion biomarkers to identify relevant and homogenous patient clusters, with a view to a more accurate predictive diagnosis and stratification for inclusion in clinical trials (Fig. 6). This heterogeneity also explains the need to use several animal models, since only one model is unable to mimic the entire clinical spectrum. Animal models should also combine both specific brain lesions and systemic vascular or metabolic disease.

The design of the preclinical and clinical studies also depends on the outcome. The integration of biomarkers

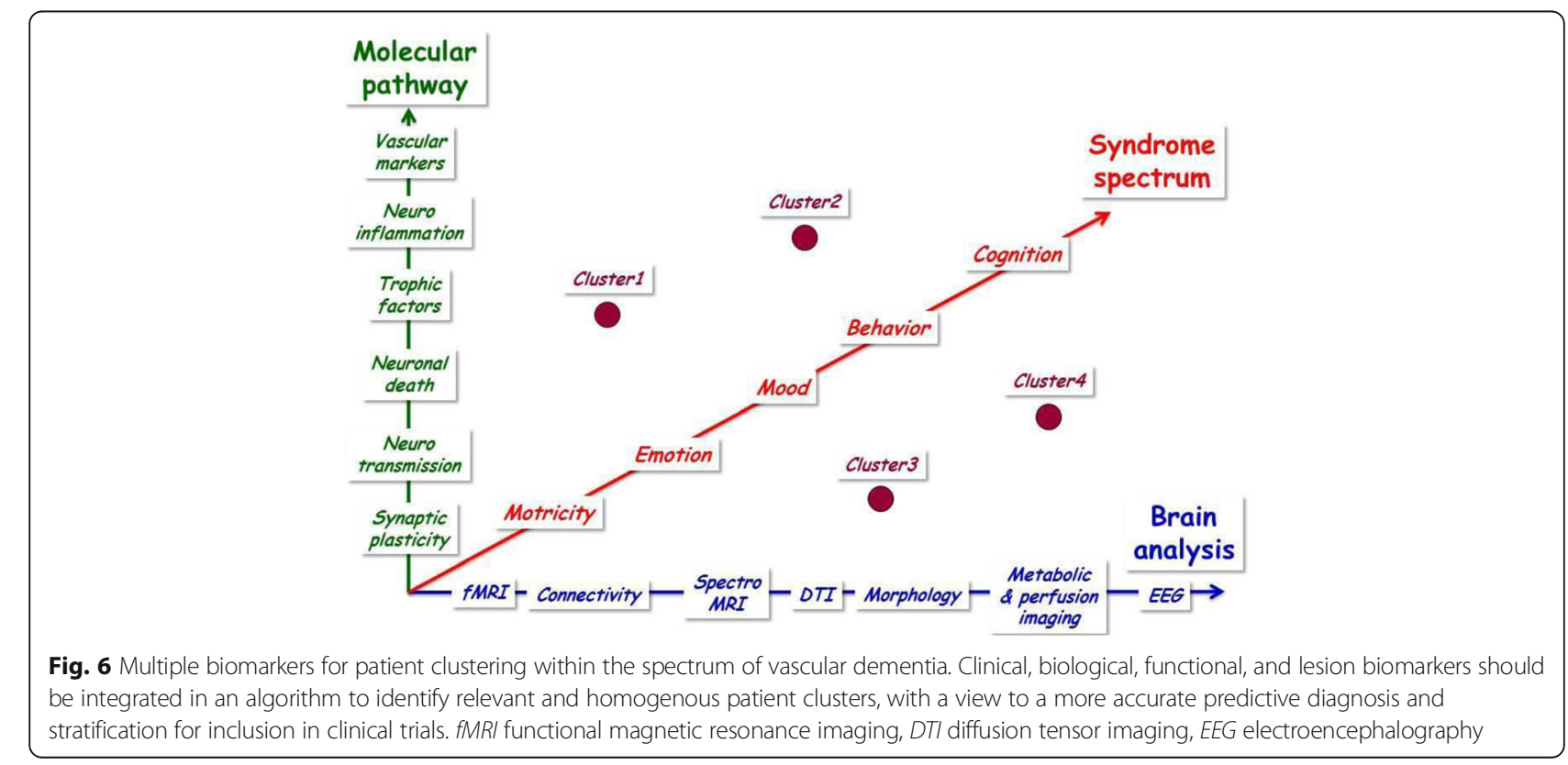


from magnetic resonance imaging (structural and functional, diffusion tensor imaging), electroencephalography, and neurophysiological markers is probably the best way to improve the assessment of drug development for the early phases $[109,110]$. Overall, these biomarkers are sensitive to both degenerative and vascular processes, allowing a more precise evaluation of brain functioning in a context of vascular impairment, where cognitive assessment remains largely unspecific. In this regard, electroencephalography cartography and multimodal analysis and sourcing of cerebral rhythms are likely to be a feasible approach to explore changes induced by hypoxia and vascular lesions in both animal models and patients. The use of these biomarkers in phase 2 might be a way to a more rapid identification of the disease-modifying strategies that should be successful in phase 3, with a stop in the process for drugs that only have an elusive impact on these biomarkers.

\section{Conclusion}

For the first time, a scientific and methodological rationale is proposed to highlight the interest of diseasemodifying strategy applications in VCI and PSCI. This proposal is based on the observation of pathophysiological substrates and clinical aspects, which are dynamic processes justifying the need of disease-modifying strategies. The second line of conclusion is that this strategy needs to be multimodal to achieve the best chance of success. Indeed, the complexity of pathophysiology explains that modulation of several targets is necessary through pleiotropic drugs or a combination with an emphasis on existing or repurposing drugs. The multimodal approach should be based on both pharmacological and non-pharmacological strategies. Finally, a specific method for the development of such an approach, in particular in the early phases, should associate (1) a better stratification of patients based on several animal models able to mimic the complete spectrum and (2) the integration of biomarkers as outcome measures to refine the assessment of relevant diseasemodifying strategies.

Funding

The consensus group was supported by Vascular Dementia Congress.

\section{Authors' contributions}

RB and AG led the consensus group and coordinated the writing of this paper. All authors contributed to the intellectual content, to the literature review, and to the writing of the first draft. All authors contributed to critical revision of the paper. All authors read and approved the final manuscript.

\section{Competing interests}

The authors declare that they have no competing interests.

\section{Publisher's Note}

Springer Nature remains neutral with regard to jurisdictional claims in published maps and institutional affiliations.

\section{Author details}

'University of Lille, Inserm, CHU, U1171 'Degenerative and vascular cognitive disorders', Lille, France. ${ }^{2}$ University of Duesseldorf, Alexian Research Center, Krefeld, Germany. ${ }^{3}$ Department of Neurology, Tel Aviv University, Ramat Aviv, Israel. ${ }^{4}$ Department of Neurology IC, Oasi Institute for Research on Mental Retardation and Brain Aging (IRCCS), Troina, Italy. ${ }^{5}$ University Medical Centre Ljubljana, Neurologic Hospital, Neurorehabilitation Unit, Ljubljana, Slovenia. ${ }^{6}$ Dr. Willmar Schwabe GmbH \& Co. KG, Karlsruhe, Germany. ${ }^{7}$ Department of Neurology, Neurosurgery and Genetics, Russian National Research Medical University, Moscow Research and Clinical Center for Neuropsychiatry, Moscow, Russia. ${ }^{8}$ Département de Pharmacologie Médicale, Faculté de Médecine, 1 place Verdun, 59045 Lille Cedex, France.

Received: 11 November 2016 Accepted: 6 May 2017

Published online: 24 May 2017

\section{References}

1. Kapasi A, Schneider JA. Vascular contributions to cognitive impairment, clinical Alzheimer's disease, and dementia in older persons. Biochim Biophys Acta. 1862;2016:878-86.

2. O'Brien JT, Erkinjuntti T, Reisberg B, Roman G, Sawada T, Pantoni L, Bowler JV, Ballard C, DeCarli C, Gorelick PB, Rockwood K, Burns A, Gauthier S, DeKosky ST. Vascular cognitive impairment. Lancet Neurol. 2003;2:89-98.

3. Korczyn AD. The complex nosological concept of vascular dementia. J Neurol Sci. 2002;203-204:3-6.

4. Akinyemi RO, Mukaetova-Ladinska EB, Attems J, Ihara M, Kalaria RN. Vascular risk factors and neurodegeneration in ageing related dementias: Alzheimer's disease and vascular dementia. Curr Alzheimer Res. 2013;10:642-53.

5. Solfrizzi V, Scafato E, Frisardi V, Seripa D, Logroscino G, Maggi S, Imbimbo BP, Galluzzo L, Baldereschi M, Gandin C, Di Carlo A, Inzitari D, Crepaldi G, Pilotto A, Panza F, Italian Longitudinal Study on Aging Working Group. Frailty syndrome and the risk of vascular dementia: the Italian Longitudinal Study on Aging. Alzheimers Dement. 2013;9:113-22.

6. Leys D, Henon H, Mackowiak-Cordoliani MA, Pasquier F. Poststroke dementia. Lancet Neurol. 2005;4:752-9.

7. Brainin M, Tuomilehto J, Heiss WD, Bornstein NM, Bath PM, Teuschl Y, Richard E, Guekht A, Quinn T. Post-stroke cognitive decline: an update and perspectives for clinical research. Eur J Neurol. 2015;22:229-38.

8. Shim H. Vascular cognitive impairment and post-stroke cognitive deficits. Curr Neurol Neurosci Rep. 2014;14:418.

9. Solfrizzi V, Scafato E, Seripa D, Lozupone M, Imbimbo BP, D'Amato A, Tortelli R, Schilardi A, Galluzzo L, Gandin C, Baldereschi M, Di Carlo A, Inzitari D, Daniele A, Sabbà C, Logroscino G, Panza F, Italian Longitudinal Study on Aging Working Group. Reversible cognitive frailty, dementia, and all-cause mortality. The Italian Longitudinal Study on Aging. J Am Med Dir Assoc. 2017;18:89.e1-8.

10. O'Brien JT, Thomas A. Vascular dementia. Lancet. 2015;386:1698-706.

11. Venkat $P$, Chopp M, Chen J. Models and mechanisms of vascular dementia. Exp Neurol. 2015;272:97-108

12. Jellinger KA. Pathology and pathogenesis of vascular cognitive impairmenta critical update. Front Aging Neurosci. 2013;5:17.

13. Korczyn AD. Why have we failed to cure Alzheimer's disease? J Alzheimers Dis. 2012;29:275-82.

14. Doody RS, Raman R, Farlow M, Iwatsubo T, Vellas B, Joffe S, Kieburtz K, He F, Sun X, Thomas RG, Aisen PS, for the Alzheimer's Disease Cooperative Study Steering Committee, Siemers E, Sethuraman G, Mohs R, for the Semagacestat Study Group. A phase 3 trial of semagacestat for treatment of Alzheimer's disease. N Engl J Med. 2013;369:341-50.

15. Ossenkoppele R, Tolboom N, Foster-Dingley JC, Adriaanse SF, Boellaard R, Yaqub M, Windhorst AD, Barkhof F, Lammertsma AA, Scheltens $P$, van der Flier WM, van Berckel BN. Longitudinal imaging of Alzheimer pathology using [11C]PIB, [18 F]FDDNP and [18 F]FDG PET. Eur J Nucl Med Mol Imaging. 2012;39:990-1000.

16. Liu E, Schmidt ME, Margolin R, Sperling R, Koeppe R, Mason NS, Klunk WE, Mathis CA, Fox NC, Hill DL, Les AS, Collins P, Gregg KM, Di J, Lu Y, Tudor IC, Wyman BT, Booth K, Broome S, Yuen E, Grundman M, Brashear HR, fort the Bapineuzumab 301 and 302 Clinical Trial Investigators. Amyloid- $\beta 11 C-P i B-$ PET imaging results from 2 randomized bapineuzumab phase 3 AD trials. Neurology. 2015;85:692-700.

17. Monsell SE, Kukull WA, Roher AE, Maarouf CL, Serrano G, Beach TG, Caselli RJ, Montine TJ, Reiman EM. Characterizing apolipoprotein E \&4 carriers and 
noncarriers with the clinical diagnosis of mild to moderate Alzheimer dementia and minimal $\beta$-amyloid peptide plaques. JAMA Neurol. 2015;72:1124-31.

18. Winblad B, Gauthier S, Scinto L, Feldman H, Wilcock GK, Truyen L, Mayorga AJ, Wang D, Brashear HR, Nye JS, for the GAL-INT-11/18 Study Group. Safety and efficacy of galantamine in subjects with mild cognitive impairment. Neurology. 2008;70:2024-35.

19. Vellas B, Bateman R, Blennow K, Frisoni G, Johnson K, Katz R, Langbaum J, Marson D, Sperling R, Wessels A, Salloway S, Doody R, Aisen P, and Task Force Members. Endpoints for pre-dementia AD trials: a report from the EU/ US/CTAD Task Force. J Prev Alzheimers Dis. 2015;2:128-35.

20. Pendlebury ST, Rothwell PM. Prevalence, incidence, and factors associated with pre-stroke and post-stroke dementia: a systematic review and metaanalysis. Lancet Neurol. 2009;8:1006-18.

21. Pohjasvaara T, Erkinjuntti T, Vataja R, Kaste M. Dementia three months after stroke. Baseline frequency and effect of different definitions of dementia in the Helsinki Stroke Aging Memory Study (SAM) cohort. Stroke. 1997;28:785-92.

22. Cordonnier C, Leys D, Dumont F, et al. What are the causes of pre-existing dementia in patients with intracerebral haemorrhages? Brain. 2010;133:3281-9.

23. Pendlebury ST, Rothwell PM. Risk of recurrent stroke, other vascular events and dementia after transient ischaemic attack and stroke. Cerebrovasc Dis. 2009;27 Suppl 3:1-11.

24. Sun JH, Tan L, Wang HF, Tan MS, Tan L, Li JQ, Xu W, Zhu XC, Jiang T, Yu JT. Genetics of vascular dementia: systematic review and meta-analysis. J Alzheimers Dis. 2015;46:611-29.

25. Hainsworth $\mathrm{AH}$, Brittain JF, Khatun $\mathrm{H}$. Pre-clinical models of human cerebral small vessel disease: utility for clinical application. J Neurol Sci. 2012;322:237-40.

26. Bink DI, Ritz K, Aronica E, van der Weerd L, Daemen MJ. Mouse models to study the effect of cardiovascular risk factors on brain structure and cognition. J Cereb Blood Flow Metab. 2013;33:1666-84.

27. Hainsworth AH, Allan SM, Boltze J, Cunningham C, Farris C, Head E, Ihara M, Isaacs JD, Kalaria RN, Lesnik Oberstein SA, Moss MB, Nitzsche B, Rosenberg GA, Rutten JW, Salkovic-Petrisic M, Troen AM. Translational models for vascular cognitive impairment: a review including larger species. BMC Med. 2017;15:16.

28. METACOHORTS Consortium. METACOHORTS for the study of vascular disease and its contribution to cognitive decline and neurodegeneration: An initiative of the Joint Programme for Neurodegenerative Disease Research. Alzheimers Dement. 2016;12(12):1235-49.

29. Strokog consortium. STROKOG (stroke and cognition consortium): An international consortium to examine the epidemiology, diagnosis, and treatment of neurocognitive disorders in relation to cerebrovascular disease. Alzheimers Dement. 2016;7:11-23.

30. Wollenweber FA, Zietemann V, Rominger A, Opherk C, Bayer-Karpinska A, Gschwendtner A, Coloma Andrews L, Bürger K, Duering M, Dichgans M. The Determinants of Dementia After Stroke (DEDEMAS) Study: protocol and pilot data. Int J Stroke. 2014;9:387-92.

31. Ben Assayag E, Korczyn AD, Giladi N, Goldbourt U, Berliner AS, ShenharTsarfaty S, Kliper E, Hallevi H, Shopin L, Hendler T, Baashat DB, Aizenstein O, Soreq H, Katz N, Solomon Z, Mike A, Usher S, Hausdorff JM, Auriel E, Shapira I, Bornstein NM. Predictors for poststroke outcomes: the Tel Aviv Brain Acute Stroke Cohort (TABASCO) study protocol. Int I Stroke. 2012;7:341-7.

32. Kalaria RN. Vascular basis for brain degeneration: faltering controls and risk factors for dementia. Nutr Rev. 2010;68 Suppl 2:S74-87.

33. Iadecola C. The pathobiology of vascular dementia. Neuron. 2013;80:844-66.

34. Le Thuc O, Blondeau N, Nahon JL, Rovère C. The complex contribution of chemokines to neuroinflammation: switching from beneficial to detrimental effects. Ann N Y Acad Sci. 2015;1351:127-40.

35. Cahill-Smith S, Li JM. Oxidative stress, redox signalling and endothelial dysfunction in ageing-related neurodegenerative diseases: a role of NADPH oxidase 2. Br J Clin Pharmacol. 2014;78:441-53.

36. Zlokovic BV. Neurovascular mechanisms of Alzheimer's neurodegeneration. Trends Neurosci. 2005;28:202-8.

37. Montagne A, Pa J, Zlokovic BV. Vascular plasticity and cognition during normal aging and dementia. JAMA Neurol. 2015;72:495-6.

38. Nelson AR, Sweeney MD, Sagare AP, Zlokovic BV. Neurovascular dysfunction and neurodegeneration in dementia and Alzheimer's disease. Biochim Biophys Acta. 1862;2016:887-900.

39. Zhou X, Li Y, Shi X, Ma C. An overview on therapeutics attenuating amyloid $\beta$ level in Alzheimer's disease: targeting neurotransmission, inflammation, oxidative stress and enhanced cholesterol levels. Am J Transl Res. 2016;8:246-69.
40. ladecola C. The overlap between neurodegenerative and vascular factors in the pathogenesis of dementia. Acta Neuropathol. 2010;120:287-96.

41. Dao E, Hsiung GY, Sossi V, Jacova C, Tam R, Dinelle K, Best JR, Liu-Ambrose $T$. Exploring the effects of coexisting amyloid in subcortical vascular cognitive impairment. BMC Neurol. 2015;15:197.

42. Stella F, Radanovic M, Canineu PR, Paula VJR, Forlenza OV. Anti-dementia medications: current prescriptions in clinical practice and new agents in progress. Ther Adv Drug Saf. 2015;6:151-65.

43. Teuschl Y, Matz K, Brainin M. Prevention of post-stroke cognitive decline: a review focusing on lifestyle interventions. Eur J Neurol. 2013;20:35-49.

44. Venna VR, Deplanque D, Allet C, Belarbi K, Hamdane M, Bordet R. PUFA induce antidepressant-like effects in parallel to structural and molecular changes in the hippocampus. Psychoneuroendocrinology. 2009;34:199-211.

45. Auchus AP, Brashear HR, Salloway S, Korczyn AD, De Deyn PP, GassmannMayer C, GAL-INT-26 Study Group. Galantamine treatment of vascular dementia: a randomized trial. Neurology. 2007;69:448-58.

46. Ritter A, Pillai JA. Treatment of vascular cognitive impairment. Curr Treat Options Neurol. 2015;17:367.

47. López-Valdés HE, Clarkson AN, Ao Y, Charles AC, Carmichael ST, Sofroniew MV, Brennan KC. Memantine enhances recovery from stroke. Stroke. 2014; 45:2093-100.

48. Cramer SC, Sur M, Dobkin BH, O'Brien C, Sanger TD, Trojanowski JQ, Rumsey JM, Hicks R, Cameron J, Chen D, Chen WG, Cohen LG, de Charms C, Duffy CJ, Eden GF, Fetz EE, Filart R, Freund M, Grant SJ, Haber S, Kalivas PW, Kolb B, Kramer AF, Lynch M, Mayberg HS, McQuillen PS, Nitkin R, Pascual-Leone A, Reuter-Lorenz P, Schiff N, Sharma A, Shekim L, Stryker M, Sullivan EV, Vinogradov S. Harnessing neuroplasticity for clinical applications. Brain. 2011;134:1591-609.

49. Bauduceau B, Doucet J, Bordier L, Garcia C, Dupuy O, Mayaudon H. Hypoglycaemia and dementia in diabetic patients. Diabetes Metab. 2010;36 Suppl 3:S106-11.

50. Biessels GJ, Staekenborg S, Brunner E, Brayne C, Scheltens P. Risk of dementia in diabetes mellitus: a systematic review. Lancet Neurol. 2006;5:64-74.

51. Levine DA, Langa KM. Vascular cognitive impairment: disease mechanisms and therapeutic implications. Neurotherapeutics. 2011;8:361-73.

52. Pearson-Fuhrhop KM, Cramer SC. Pharmacogenetics of neural injury recovery. Pharmacogenomics. 2013;14:1635-43.

53. Meneilly GS, Tessier DM. Diabetes, dementia and hypoglycemia. Can J Diabetes. 2016;40:73-6.

54. Bornstein NM, Brainin M, Guekht A, Skoog I, Korczyn AD. Diabetes and the brain: issues and unmet needs. Neurol Sci. 2014;35:995-1001.

55. Gorelick PB. Role of inflammation in cognitive impairment: results of observational epidemiological studies and clinical trials. Ann N Y Acad Sci. 2010;1207:155-62.

56. Patrone C, Eriksson O, Lindholm D. Diabetes drugs and neurological disorders: new views and therapeutic possibilities. Lancet Diabetes Endocrinol. 2014;2:256-62.

57. Ankolekar S, Geeganage C, Anderton P, Hogg C, Bath PM. Clinical trials for preventing post stroke cognitive impairment. J Neurol Sci. 2010;299:168-74.

58. Manktelow BN, Potter JF. Interventions in the management of serum lipids for preventing stroke recurrence. Cochrane Database Syst Rev. 2009;3, CD002091.

59. Thiel A, Cechetto DF, Heiss WD, Hachinski V, Whitehead SN. Amyloid burden, neuroinflammation, and links to cognitive decline after ischemic stroke. Stroke. 2014;45:2825-9.

60. Zhuang S, Li J, Wang X, Wang HF, Zhang WJ, Wang HY, Xing CM. Reninangiotensin system-targeting antihypertensive drugs and risk of vascular cognitive impairment: a meta-analysis. Neurosci Lett. 2016;615:1-8.

61. Ouk T, Potey C, Gautier S, Bastide M, Deplanque D, Staels B, Duriez P, Leys D, Bordet R. PPARs: a potential target for a disease-modifying strategy in stroke. Curr Drug Targets. 2013;14:752-67.

62. Floel A, Cohen LG. Recovery of function in humans: cortical stimulation and pharmacological treatments after stroke. Neurobiol Dis. 2010;37:243-51.

63. Rothman SM, Griffioen KJ, Wan R, Mattson MP. Brain-derived neurotrophic factor as a regulator of systemic and brain energy metabolism and cardiovascular health. Ann N Y Acad Sci. 2012;1264:49-63.

64. Allegri RF, Guekht A. Cerebrolysin improves symptoms and delays progression in patients with Alzheimer's disease and vascular dementia. Drugs Today (Barc). 2012;48(Suppl A):25-41.

65. Chen N, Yang M, Guo J, Zhou M, Zhu C, He L. Cerebrolysin for vascular dementia. Cochrane Database Syst Rev. 2013;1, CD008900. 
66. Guekht AB, Moessler H, Novak PH, Gusev El, Cerebrolysin Investigators. Cerebrolysin in vascular dementia: improvement of clinical outcome in randomized, double-blind, placebo-controlled multicenter trial. J Stroke Cerebrovasc Dis. 2011;20:310-8.

67. Ubhi K, Rockenstein E, Vazquez-Roque R, Mante M, Inglis C, Patrick C, Adame A, Fahnestock M, Doppler E, Novak P, Moessler H, Masliah E. Cerebrolysin modulates pronerve growth factor/nerve growth factor ratio and ameliorates the cholinergic deficit in a transgenic model of Alzheimer's disease. J Neurosci Res. 2013;91:167-77.

68. Skoog I, Korczyn AD, Guekht A. Neuroprotection in vascular dementia: a future path. J Neurol Sci. 2012;322:232-6.

69. Machicao F, Muresanu DF, Hundsberger H, Pflüger M, Guekht A. Pleiotropic neuroprotective and metabolic effects of Actovegin's mode of action. J Neurol Sci. 2012;322:222-7.

70. Meilin S, Machicao F, Elmlinger M. Treatment with Actovegin improves spatial learning and memory in rats following transient forebrain ischaemia. J Cell Mol Med. 2014;18:1623-30.

71. Federico A, Guekht A, Brainin M. Current perspectives in post-stroke cognitive impairment. European Neurological Rev. 2013:8:136-40.

72. Guekht A, Skoog I, Korczyn AD, Zakharov V, Eeg M, Vigonius U. A randomised, double-blind, placebo-controlled trial of actovegin in patients with post-stroke cognitive impairment: ARTEMIDA study design. Dement Geriatr Cogn Dis Extra. 2013;3:459-67.

73. Müller WE, Heiser J, Leuner K. Effects of the standardized Ginkgo biloba extract EGb $761^{\circledR}$ on neuroplasticity. Int Psychogeratr. 2012;24 Suppl 1:S21-4.

74. Lang F, Hoerr R, Noeldner M, Koch E. Ginkgo biloba extract EGb $761^{\circ}$ : From an ancient Asian plant to a modern European herbal medicinal product. In: Wagner H, Ulrich-Merzenich G, editors. Evidence and Rational Based Research on Chinese Drugs. Wien: Springer; 2013. p. 431-70.

75. Janssen IM, Sturtz S, Skipka G, Zentner A, Garrido MV, Busse R. Ginkgo biloba in Alzheimer's disease: a systematic review. Wien Med Wochenschr. 2010;160:539-46.

76. Weinmann S, Roll S, Schwarzbach C, Vauth C, Willich SN. Effects of Ginkgo biloba in dementia: systematic review and meta-analysis. BMC Geriatr. 2010;10:14-26.

77. von Gunten A, Schlaefke S, Uberla K. Efficacy of Ginkgo biloba extract EGb $761^{\oplus}$ in dementia with behavioural and psychological symptoms: a systematic review. World J Biol Psychiatry. 2015;27:1-12.

78. Pennisi G, Bella R, Lanza G. Motor cortex plasticity in subcortical ischemic vascular dementia: what can TMS say? Clin Neurophysiol. 2015:126:851-2.

79. Lanza G, Bramanti P, Cantone M, Pennisi M, Pennisi G, Bella R. Vascular cognitive impairment through the looking glass of transcranial magnetic stimulation. Behav Neurol. 2017;2017:1421326.

80. Elder GJ, Taylor JP. Transcranial magnetic stimulation and transcranial direct current stimulation: treatments for cognitive and neuropsychiatric symptoms in the neurodegenerative dementias? Alzheimers Res Ther. 2014;6:74.

81. Guerra A, Petrichella S, Vollero L, Ponzo D, Pasqualetti P, Määttä S, Mervaala E, Könönen M, Bressi F, lannello G, Rossini PM, Ferreri F. Neurophysiological features of motor cortex excitability and plasticity in subcortical ischemic vascular dementia: a TMS mapping study. Clin Neurophysiol. 2015;126:906-13.

82. Rektorova I, Megova S, Bares M, Rektor I. Cognitive functioning after repetitive transcranial magnetic stimulation in patients with cerebrovascular disease without dementia: a pilot study of seven patients. J Neurol Sci. 2005;229-230:157-61.

83. Brunoni AR, Benseñor IM, Alves TC. Therapeutic interventions for vascular depression: a systematic review. Rev Bras Psiquiatr. 2011;33:400-9.

84. Fritsch B, Reis J, Martinowich K, Schambra HM, Ji Y, Cohen LG, Lu B. Direct current stimulation promotes BDNF-dependent synaptic plasticity: potential implications for motor learning. Neuron. 2010;66:198-204.

85. Wang F, Chang GM, Yu Q, Geng X. The neuroprotection of repetitive transcranial magnetic stimulation pre-treatment in vascular dementia rats. J Mol Neurosci. 2015;56:198-204

86. Zeng $L$, Zou Y, Kong L, Wang N, Wang Q, Wang L, Cao Y, Wang K, Chen Y, Mi S, Zhao W, Wu H, Cheng S, Xu W, Liang W. Can Chinese herbal medicine adjunctive therapy improve outcomes of senile vascular dementia? Systematic Review with meta-analysis of clinical trials. Phytother Res. 2015;29:1843-57.

87. Feng M, Lu J, May BH, Liu S, Guo X, Zhang AL, Xue CC, Lu C. Chinese herbal medicine for patients with vascular cognitive impairment no dementia: protocol for a systematic review. BMJ Open. 2016;6, e010295.

88. Yang BF, Zeng XH, Liu Y, Fu QN, He T, Li F, Shi GX, Liu BZ, Sun SF, Wang J, Xiao L, Deng YM, Liu CZ. Effect of acupuncture treatment on vascular cognitive impairment without dementia: study protocol for a randomized controlled trial. Trials. 2014;15:442.
89. Min D, Xu-Feng W. An updated meta-analysis of the efficacy and safety of acupuncture treatment for vascular cognitive impairment without dementia. Curr Neurovasc Res. 2016;13:230-8.

90. Pinter MM, Brainin M. Rehabilitation after stroke in older people. Maturitas. 2012;71:104-8.

91. Forbes D, Forbes SC, Blake CM, Thiessen EJ, Forbes S. Exercise programs for people with dementia. Cochrane Database Syst Rev. 2015;4, CD006489.

92. Ahlskog JE, Geda YE, Graff-Radford NR, Petersen RC. Physical exercise as a preventive or disease-modifying treatment of dementia and brain aging. Mayo Clin Proc. 2011;86:876-84.

93. Voss M, Nagatasamu LS, Liu-Ambrose T, Kramer A. Exercise, brain and cognition across the life span. J Appl Physiol. 2011;111:1505-13.

94. Dunlop D, Song J, Arnston EK, Semanik PA, Lee J, Chang RW, Hootman JM. Sedentary time in U.S. older adults associated with disability in activities of daily living independent of physical activity. J Phys Act Health. 2015;12:93-101.

95. Snider J, Muller MLTM, Kotagal V, Koeppe RA, Scott PJH, Frey KA, Albin RL, Bohnen NI. Non-exercise physical activity attenuates motor symptoms in Parkinson disease independent from nigrostriatal degeneration. Parkinsonism Relat Disord. 2015;21:1227-31.

96. Jansa J, Aragon A. Living with Parkinson's and the emerging role of occupational therapy. Parkinson Dis. 2015:196303.

97. Law M, Cooper B, Strong S, Stewart D, Rigby P, Letts L. The personenvironment-occupation model: a transactive approach to occupational performance. Can J Occup Ther. 1996;63:9-23.

98. Bouwens SFM, van Heugten C, Aalten P, Wolfs CAG, Baarends EM, van Menxel DAJ, Verhey FRJ. Relationship between measures of dementia severity and observation of daily life functioning as measured with the Assessment of Motor and Process Skills (AMPS). Dement Geriatr Cogn Disord. 2008;25:81-7.

99. Graff MJ, Vernooij-Dassen MJ, Thijssen M, Dekker J, Hoefnagels WH, OldeRikkert MG. Community occupational therapy in patients with dementia and their caregivers: a randomized controlled trial. BMJ. 2008:336:134-8.

100. Spruytte N, van Audenhove C, Lammertyn F. Predictors of institutionalization of cognitively-impaired elderly cared for by their relatives. Int J Geriatr Psychiatry. 2001;16:1119-28.

101. Guichen L, Hua Y, Wei Z. Effects of Tai Chi on health related quality of life in patients with chronic conditions: A systematic review of randomized controlled trials. Compliment Ther Med. 2014;22:743-55.

102. Ueda T, Suzukamo Y, Sato M, Izumi SI. Effects of music therapy on behavioural and psychological symptoms of dementia: a systematic review and meta-analysis. Ageing Res Rev. 2013;12:628-41.

103. Ihl R, Frölich L, Winblad B, Schneider L, Burns A, Möller HJ, WFSBP Task Force on Treatment Guidelines for Alzheimer's Disease and other Dementias. World Federation of Societies of Biological Psychiatry (WFSBP) guidelines for the biological treatment of Alzheimer's disease and other dementias. World J Biol Psychiatry. 2011;12:2-32.

104. Weuve J, Proust-Lima C, Power MC, Gross AL, Hofer SM, Thiébaut R, Chêne G, Glymour MM, Dufouil C, MELODEM Initiative. Guidelines for reporting methodological challenges and evaluating potential bias in dementia research. Alzheimers Dement. 2015;11:1098-109.

105. Rosenthal R, DiMatteo MR. Meta-analysis: recent developments in quantitative methods for literature reviews. Annu Rev Psychol. 2001;52:59-82.

106. Schroter S, Black N, Evans S, Godlee F, Osorio L, Smith R. What errors do peer reviewers detect, and does training improve their ability to detect them? J R Soc Med. 2008;101:507-14.

107. Smith GC, Pell JP. Parachute use to prevent death and major trauma related to gravitational challenge: systematic review of randomised controlled trials. BMJ. 2003;327:1459-61.

108. Nelson KS, Brearley AM, Haines SJ. Evidence-based assessment of wellestablished interventions: the parachute and the epidural hematoma. Neurosurgery. 2014;75:552-9.

109. Deguil J, Ravasi L, Auffret A, Babiloni C, Bartres Faz D, Bragulat V, Cassé-Perrot C, Colavito V, Herrero Ezquerro MT, Lamberty Y, Lanteaume L, Pemberton D, Pifferi F, Richardson JC, Schenker E, Blin O, Tarragon E, Bordet R. Evaluation of symptomatic drug effects in Alzheimer's disease: strategies for prediction of efficacy in humans. Drug Discov Today Technol. 2013;10:329-42.

110. Deguil J, Ravasi L, Lamberty Y, Auffret A, Payoux P, Leroy C, Derambure P, Bordet R. Early Development of Symptomatic Drugs in AD: A Systematic Review of the Use of Biomarkers. CNS Neurol Disord Drug Targets. 2016;15:823-36. 